\title{
Balanço de energia e coeficiente de cultura da cana-de-açúcar por dois métodos micrometeorológicos
}

\author{
José Francisco Alves do Carmo ${ }^{1}$, Magna Soelma Beserra de Moura ${ }^{2(*)}$, Thieres George Freire da Silva ${ }^{3}$, \\ Luciana Sandra Bastos de Souza ${ }^{3}$, Mario de Miranda Vilas Boas Ramos Leitao ${ }^{4}$ \\ ${ }^{1}$ Mestre, CPGEA, UNIVASF, Juazeiro-BA, j.francarmo@gmail.com \\ ${ }^{2}$ Pesquisadora, Embrapa Semiárido, Petrolina-PE, magnamoura@embrapa.br \\ ${ }^{3}$ Professor, UFRPE/UAST, Serra Talhada-PE, thieres_freire@yahoo.com.br, sanddrabastos@yahoo.com.br; 4Professor, PPGEA, UNIVASF, Juazeiro-BA, \\ mario.miranda@univasf.edu.br \\ ${ }^{(*)}$ Autor para correspondência
}

\section{INFORMAÇÕES}

\section{História do artigo:}

Recebido em 16 de Junho de 2017

Aceito em 10 de agosto de 2017

Termos para indexação:

correlações turbulentas

evapotranspiração

razão de Bowen

Vale do São Francisco

Saccharum spp

\section{RESUMO}

Objetivou-se analisar os componentes do balanço de energia obtidos pelos métodos das correlações turbulentas (EC) e da razão de Bowen (BERB), e determinar a evapotranspiração e o coeficiente de cultura da cana-de-açúcar irrigada por gotejamento subsuperficial sob as condições climáticas do Submédio do Vale do São Francisco. Para tanto, densidades de fluxos de calor latente e sensível foram medidas sobre um cultivo ao longo do ciclo de produção durante o período de novembro de 2010 a outubro de 2011. Com os resultados obtidos foi possível verificar que o método EC apresentou subestimativa da energia disponível em $26 \%$, e o fluxo de calor latente em 19\% em relação ao método da Razão de Bowen. Os valores diários da fração evaporativa variaram entre 0,66 (método das correlações turbulentas) e 0,68 (método da Razão de Bowen), revelando que a maior parte da energia disponível foi convertida em evapotranspiração real. A evapotranspiração média diária da cultura diferiu entre os dois métodos, apresentando valores de 3,6 e 4,1 $\mathrm{mm} \mathrm{d}^{-1}$ para os métodos EC e BERB, resultando em um requerimento hídrico total de $1478 \mathrm{~mm}$. Os valores do coeficiente de cultura da cana-de-açúcar obtidos foram 0,54 e 075 na fase inicial, 0,83 e 0,87 na fase de máximo crescimento para os métodos EC e BERB, respectivamente, e 0,52 para a fase final, determinado pelo método BERB.

(c) 2017 SBAgro. Todos os direitos reservados.

\section{Introdução}

O uso adequado da água no sistema de produção agrícola pode ser controlado por meio do conhecimento dos requerimentos hídricos das culturas, dentre os quais é muito importante a determinação da evapotranspiração (ET). A ET é um dos principais componentes do ciclo hidrológico, sendo um consumidor primário de enormes volumes de água (ALLEN et al., 2011).

A determinação da ET é bastante relevante para assegurar o suprimento de água, bem como para diminuir os impactos ambientais da irrigação e melhorar o cálculo das perdas de água por drenagem e escoamento em cultivos de cana-de-açúcar, os quais são frequentemente superdimen- 
sionados (INMAN BAMBER; MCGLINCHEY, 2003; INMAN BAMBER; SMITH, 2005; SILVA et al., 2012).

Em áreas irrigadas, a estimativa da evapotranspiração das culturas (ETc) pode ser obtida pela partição da energia disponível (Rn-G) entre calor sensível (H) e calor latente $(\lambda \mathrm{E})$, utilizando o método do balanço de energia com base na razão de Bowen (BERB), que se baseia na relação do fluxo-gradiente e transferência de massa e pelo método das correlações turbulentas ou "Eddy Covariance" (EC). Esses métodos têm sido amplamente aplicados em culturas como milho (STEDUTO; HSIAO, 1998), café (MARIN, 2003), forrageira (SILVA et al., 2005), videira (TEIXEIRA et al., 2007), cana-de-açúcar (SILVA et al., 2011b; SILVA et al., 2012), manga (TEIXEIRA; BASTIAANSSEN, 2012), entre outras. Por meio da razão ETc/ETo (sendo ETo a evapotranspiração de referência), obtêm-se o coeficiente de cultivo (Kc), que varia de acordo com os estádios fenológicos da cultura e que é utilizado no manejo de irrigação.

Assim, objetivou-se analisar os componentes do balanço de energia mensurados pelos métodos das correlações turbulentas e da razão de Bowen, e a evapotranspiração e o coeficiente de cultura da cana-de-açúcar irrigada por gotejamento subsuperficial, nas condições climáticas do Submédio do Vale do São Francisco.

\section{Material e métodos}

A pesquisa foi conduzida em um campo de produção de cana-de-açúcar da Usina Agroindústrias do Vale do São Francisco - AGROVALE (latitude: 9²9'S; longitude: 402ㄴ'O; altitude: $395 \mathrm{~m}$ ). O clima da região, de acordo com a classificação de Koppen, é do tipo BSwh'.

O campo de produção de cana-de-açúcar selecionado para instalação deste estudo compreendeu uma área de 7,86 ha, cultivado com a variedade RB 92-579, em Vertissolo, em espaçamento duplo de $1,3 \times 0,7 \mathrm{~m}$, irrigada por gotejamento subsuperficial, com gotejadores enterrados a 0,20 $\mathrm{cm}$ da superfície do solo. O estudo foi realizado para o ciclo de cana-soca, que compreendeu o período de 18 de novembro de 2010 a 31 de outubro de 2011, totalizando 347 dias.

No centro do campo foi instalada uma torre micrometeorológica equipada com sensores para medir os elementos meteorológicos e o balanço de energia. Para a obtenção dos componentes do balanço de energia foram instalados dois sistemas, sendo com medidas em baixa frequência, para uso da metodologia do Balanço de Energia com Base na Razão de Bowen (BERB) e outro em alta frequência, para utilização do método das correlações turbulentas ou Eddy Covariance (EC).

O balanço de energia $(R N)$ simplificado no sistema solo-planta-atmosfera é constituído pelos fluxos verticais de calor latente $(\lambda \mathrm{E})$, calor sensível $(H)$, calor no solo $(G)$, todos expressos em $\mathrm{W} \mathrm{m}^{-2}$. Assim, o balanço de energia sim- plificado à superfície se reduz à seguinte equação:

$$
R n=\lambda E+H+G
$$

O saldo de radiação $(R n)$ foi medido por meio de um saldo radiômetro, modelo CNR1 (Net radiometer - Kipp \& Zonen B.V., Delft, Netherlands), instalado a 4,0 m acima do dossel da cultura, enquanto o fluxo de calor no solo $(G)$, por dois fluxímetros (HFT3-REBS, Campbell Scientific, Inc. Logan, Utah, USA) colocados inter e entre fileiras de plantas a 0,06 $\mathrm{m}$ de profundidade. Além destes, foram feitas medições da temperatura e da umidade relativa do ar utilizando um sensor HMP45C (Vaisala, Finlândia), velocidade e direção do vento medidos com um anemômetro (03001-5, R. M. Young, Wind Sentry, Campbell Scientific, Inc. Logan, Utah, USA) e da precipitação pluviométrica, por meio de um pluviômetro (CS700-L Hydrological Services Rain Gage, Liverpool, Austrália). Para armazenamento dos dados em baixa frequência foi utilizado um datalogger modelo CR23X (Campbell Scientific, Inc. Logan, Utah, USA) programado para realizar medidas de30 s e armazenar médias a cada 30 min.

Pelo método da razão de Bowen, os fluxos de $H$ e $\lambda \mathrm{E}$ foram obtidos por:

$$
\begin{gathered}
\lambda E_{R B}=\frac{R n-G}{1+\beta} \\
H_{R B}=\frac{\beta(R n-G)}{1+\beta}
\end{gathered}
$$

em que $R n, H, \lambda \mathrm{E}$ e $G$ e são dados em $\mathrm{W} \mathrm{m} \mathrm{m}^{-2}$ e $\beta$, que corresponde a razão de Bowen, é adimensional, e pode ser calculado pela expressão:

$$
\beta=\gamma\left(\frac{\Delta t}{\Delta e}\right)
$$

em que $\Delta$ t e $\Delta e$ são as diferenças de temperatura do ar $\left({ }^{\circ} \mathrm{C}\right)$ e de pressão de vapor d'água $(\mathrm{kPa})$, respectivamente. Para sua obtenção foram realizadas medidas da temperatura de bulbo seco $\left(t_{b s}\right)$ e de bulbo úmido $\left(\left(t_{b u}\right)\right.$ em psicrômetros situados nos níveis $Z_{1}$ e $Z_{2}$ acima do dossel, sendo o primeiro sempre ajustado à altura de 1,5 vezes a altura média da cultura, conforme proposto por Inman Bamber e McGlinchey (2003) e o segundo a 1,0 m acima do primeiro. Assim, considerando a equação psicrométrica, os valore de $\beta$ foram dados por: 


$$
\beta=\left[\frac{\Delta+\gamma}{\gamma} \frac{\left(t_{b u 1}-t_{b u 2}\right)}{\left(t_{b s 1}-t_{b s 2}\right)}-1\right]
$$

em que $\Delta$ é a declividade da curva de pressão de vapor d'água $\left(\mathrm{kPa}{ }^{\circ} \mathrm{C}^{-1}\right), T_{b u 1}, T_{b u 2}$ e $T_{b s 1}$ são as temperaturas de bulbo úmido e seco $\left({ }^{\circ} \mathrm{C}\right)$ correspondentes aos níveis $Z_{1}$ e $Z_{2}$.

Os erros associados ao método BERB na estimativa de $\beta$, e consequentemente no cálculo de $\lambda E$ foram avaliados seguindo os critérios definidos por Perez et al. (1999), com base nos dados do perfil de pressão de vapor d'água em cada horário de medição, na resolução dos sensores e na conversão de sinais da relação fluxo-gradiente.

Os fluxos de $\lambda E$ e $H$ pelo método de $E C$ foram medidos a partir de um sistema composto por um higrômetro modelo KRYPTON KH $\mathrm{H}_{2} \mathrm{O}$ (Campbell Scientific, Logan, Utah, USA) para mensuração das concentrações de vapor de água; um anemômetro sônico tridimensional modelo CSAT3 (Campbell Scientific, Logan, Utah, USA) para medição da tempen ratura do ar (T) e os componentes da velocidade do vento nas direções X, y e z, e por um sensor HMP45C (Vaisala, Finlândia), para medir a temperatura e a umidade relativa do ar. Os equipamentos foram fixados $8,0 \mathrm{~m}$ acima da altura média da vegetação da área de estudo, posicionados considerando a direção do vento predominante. As medidas foram realizadas à taxa de $10 \mathrm{~Hz}$, por meio de um datalogger modelo CR1000 (Campbell Scientific Inc. Logan, UT, EUA).

A partir dos dados de covariância, os fluxos de $\lambda E$ e de $H$ foram calculados para intervalos de 30 minutos, segundo as seguintes expressões:

$$
\begin{gathered}
\lambda E_{E C}=\lambda \overline{w^{\prime} \rho_{v}{ }^{\prime}} \\
H_{E C}=\rho_{a} c_{p} \overline{w^{\prime} T_{a}{ }^{\prime}}
\end{gathered}
$$

em que $\lambda E_{E C}$ e $H_{E C}$ são expressos em $\mathrm{W} \mathrm{m}^{-2} ; \lambda$ é o calor latente de vaporização $\left(\mathrm{J} \mathrm{Kg}^{-1}\right)$; w', $P_{v^{\prime}}$ e $T_{a^{\prime}}$ são os desvios instantâneos da velocidade vertical do vento $\left(\mathrm{m} \mathrm{s}^{-1}\right)$, da densidade de vapor d'água $\left(\mathrm{Kg} \mathrm{m}^{-3}\right)$ e temperatura do ar $\left({ }^{\circ} \mathrm{C}\right)$, respectivamente, em relação aos valores médios; $P_{a}$ é a densidade do $\operatorname{ar}\left(\mathrm{Kg} \mathrm{m}^{-3}\right) ; C_{p}$ é o calor específico do ar à pressão constante $\left(\mathrm{J} \mathrm{Kg}^{-1} \mathrm{~K}^{-1}\right)$.

Para correções de erros e análise de consistência dos dados de alta frequência, bem como para as correções dos erros inerentes ao problema do "não fechamento" do balanço de energia pelo método de EC, foram utilizados as metodologias descritas em Teixeira e Bastiaanssen (2012).

Para converter os valores do fluxo, que representa a energia por unidade de área e por unidade de tempo, em unidade de lâmina evaporada e, ou, transpirada em um dado intervalo de tempo, ou seja, a evapotranspiração atual (ETa) foi utilizada a relação entre $\lambda E$ e $\lambda$ multiplica- do pelo intervalo de tempo das medições correspondentes ao saldo de radiação, gradientes de temperatura e de vapor d'água e do fluxo de calor no solo, logo:

$$
E T a=\frac{\lambda E . i t . f_{\text {tempo }}}{\lambda}
$$

em que $\lambda \mathrm{E}$ e $\lambda$ são expressos em $\mathrm{W} \mathrm{m}^{-2}$; it é o intervalo de tempo armazenamento dos valores médios das medidas (30 min); ftempo é o fator de ajuste da escala de tempo (60 segundos). Posteriormente, foi realizada a integração destes valores para obtenção da diária da cultura $\left(\mathrm{mm} \mathrm{dia}^{-1}\right)$, como segue:

$$
E T=\sum_{R n-G>0}^{i=1} E T a
$$

Com base na eq. 6, foi calculado o requerimento hídrico da cultura pela soma dos valores diários de ET obtidos no período de monitoramento, correspondentes a cada fase fenológica da cana-de-açúcar, delimitadas como: Fase I ( $F_{1}$ - brotação e estabelecimento), Fase II ( $F_{2}$ - estabelecimento e perfilhamento), Fase III ( $F_{3}$ - crescimento máximo) e Fase IV ( $F_{4}$ - maturação fisiológica).

A evapotranspiração de referência foi calculada por meio de dados diários de radiação solar global $(\mathrm{Qg})$, temperatura do ar $\left(T_{\alpha}\right)$, precipitação $(P)$, umidade relativa do ar (UR) e velocidade do vento (Vv) de uma estação agrometeorológica automática instalada em uma área próxima à Usina, na Fazenda Brasil Uvas. Para o cálculo da ETo, utilizou-se o método de Penman Monteith padronizado pela FAO (ALLEN et al., 1998), dado pela expressão:

$$
E T o=\frac{0,408 \Delta(R n-G)+\gamma \frac{900}{T+273} u_{2}\left(e_{s}-e_{a}\right)}{\Delta+\gamma\left(1+0,34 u_{2}\right)}
$$

Com base na razão entre os valores de ET e ETo, foi determinado o coeficiente de cultivo $(K c)$ tanto a nível diário, como para as diferentes fases de crescimento da cultura, segundo a expressão:

$$
K c=\frac{E T}{E T o}
$$

onde, Kc é adimensional e ETc e ETo são expressos em mm.dia ${ }^{-1}$. 
Tabela 1. Análise de consistência física dos dados da razão de Bowen ( $\beta$ ), Juazeiro-BA.

\begin{tabular}{|c|c|c|c|c|c|c|c|c|}
\hline \multirow{2}{*}{$\begin{array}{l}\text { Período (no } \\
\text { de dias) }\end{array}$} & \multirow{2}{*}{$\begin{array}{c}\text { Dados } \\
\text { consistent } \\
\text { es }(\%)^{*}\end{array}$} & \multicolumn{4}{|c|}{ Tipos de erro } & \multirow{2}{*}{$\begin{array}{c}\beta<-0,75 \\
*\end{array}$} & \multirow{2}{*}{$\begin{array}{c}(-1-|\varepsilon|) \\
<\beta< \\
(-1+|\varepsilon|) *\end{array}$} & \multirow{2}{*}{$\begin{array}{c}\text { Dias } \\
\text { válidos } \\
(\%)^{* *}\end{array}$} \\
\hline & & $A^{*}$ & $B^{*}$ & $C^{*}$ & $D *$ & & & \\
\hline $\begin{array}{c}F 1 \\
(10 \text { dias })\end{array}$ & 2,1 & 0,0 & 0,1 & 0,5 & 0,0 & 0,5 & 0,7 & 100 \\
\hline $\begin{array}{c}F 2 \\
\text { (89 dias) }\end{array}$ & 13,4 & 0,0 & 0,2 & 11,0 & 0,0 & 2,9 & 6,6 & 89,9 \\
\hline $\begin{array}{c}F 3 \\
\text { (195 dias) }\end{array}$ & 35,9 & 0,0 & 4,3 & 13,1 & 0,0 & 7,1 & 11,4 & 74,4 \\
\hline $\begin{array}{c}\mathrm{F} 4 \\
\text { (29 dias) }\end{array}$ & 1,8 & 0,0 & 0,1 & 0,4 & 0,0 & 0,8 & 0,5 & 50,0 \\
\hline Ciclo & 56,8 & 0,0 & 5,8 & 25,6 & 0,0 & 11,8 & 19,2 & 77,4 \\
\hline
\end{tabular}

Obs.: *Considerando o numero total de dados observados (15504 dados); **Considerando o número de dias monitorados (323 dias); Tipos de erro: "A" $=(R n-G)>0 ; \Delta e>0 ; \beta<-1+|\varepsilon|$; "B" $=(\mathrm{Rn}-\mathrm{G})>0 ; \Delta \mathrm{e}<0 ; \beta>-1-|\varepsilon|$; "C" $=(\mathrm{Rn}-\mathrm{G})<0 ; \Delta \mathrm{e}>0 ; \beta>-1-|\varepsilon| ; " \mathrm{D} "=(\mathrm{Rn}-\mathrm{G})<0 ; \Delta \mathrm{e}<0 ; \beta<-1+|\varepsilon|$

\section{Resultados e discussão}

$\mathrm{Na}$ análise da consistência física dos dados da razão de Bowen $(\beta)$ foi analisado um total de 15504 dados médios de intervalos de $30 \mathrm{~min}$ ao longo do ciclo da cultura, revelando que $56,8 \%$ deles foram considerados fisicamente consistentes (Tabela 1).

Após a análise de consistência dos dados e assumindo como dias válidos aqueles que apresentaram, pelo menos, $80 \%$ dos dados consistentes no período diurno (Silva et al., 2011), observou-se que a fase $F_{1}$ não apresentou dias inválidos, enquanto a $F_{4}$ destacou-se como a mais afetada, tendo $50 \%$ dos dias sido considerados inválidos (Tabela 1 ). A fase $F_{3}$ apresentou os menores valores de $\beta$, bem como maior número de dias considerados inválidos (50 dias), representando $68,5 \%$ do total de dias rejeitados. Isso se deve ao tombamento característico da cana, que pode ocorrer nessa fase fenológica, após os 180 DAC, o que implica no aumento da distância entre o dossel da cultura e os psicrômetros do sistema BERB, reduzindo de forma bastante expressiva o gradiente de temperatura do ar $\left(\Delta t_{b s}\right)$ e, em menor expressão, o gradiente da temperatura de bulbo úmido $\left(\Delta \mathrm{t}_{\mathrm{bu}}\right)$. Em termos de ciclo, do total de 323 dias monitorados 77,4\% (250 dias) foram considerados válidos para estimativa dos fluxos de $L E$ e $H$.

O fechamento do balanço de energia pelo método EC durante o período de estudo foi realizado em escala diária (Figura 1). Nessa escala de tempo, os erros concernentes às medições do fluxo de calor no solo atribuídos às variações da umidade do solo e nível de sombreamento são reduzidos. O valor médio da razão entre $(H+\lambda E)_{E C}$ e $(R n-G)$ foi de $74,0 \%$ (a), com uma raiz do erro médio quadrático (RMSE) de 4,0 MJ m-2 $\mathrm{d}^{-1}$, que correspondeu a $27,2 \%$ da energia disponível.

Teixeira e Bastiaansen (2012) encontraram RMSE de 1,7

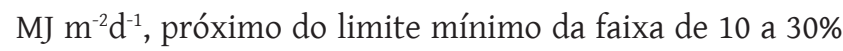
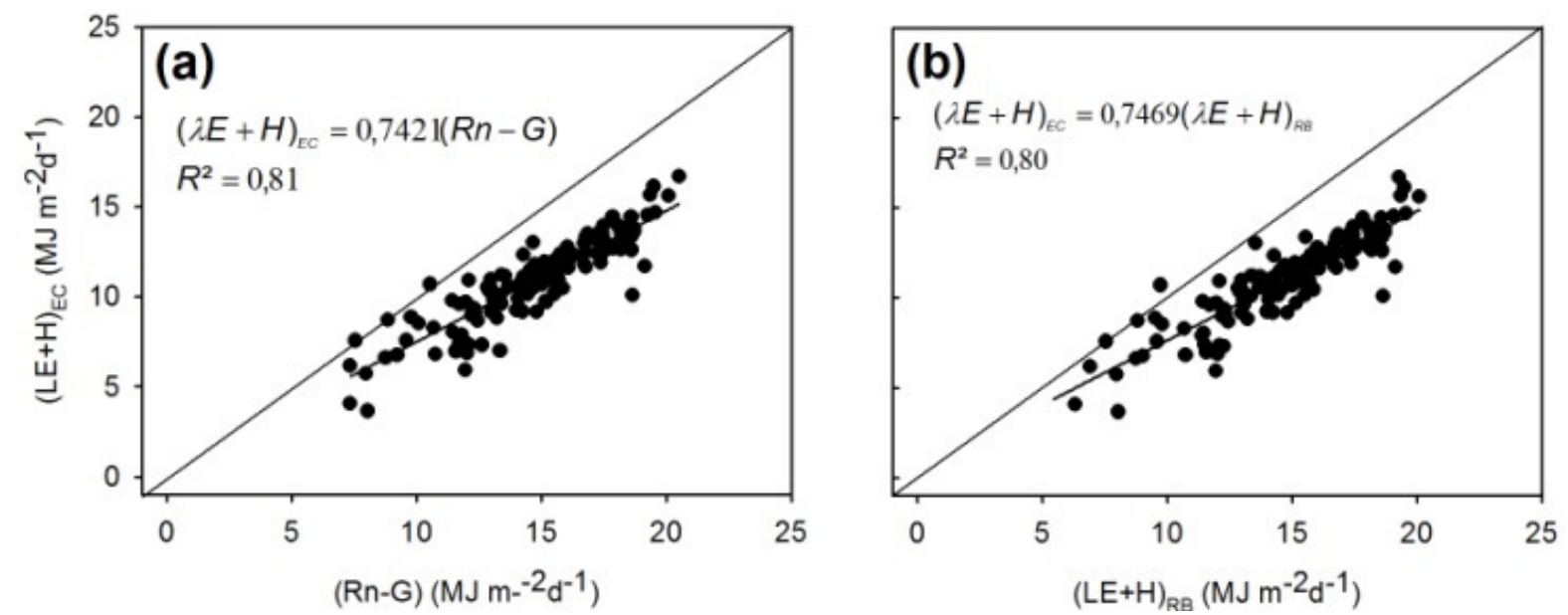

Figura 1. Relação entre a soma dos fluxos de calor latente e sensível determinado pelo método das correlações turbulentas $(+H)_{E C}$ com a energia disponível (Rn-G) (a) e com a soma dos fluxos de calor latente e sensível determinado pelo método da razão de Bowen $(+H)_{R B}$ para a cana-de-açúcar irrigada por gotejamento subsuperficial no Submédio do Vale São Francisco, Juazeiro-BA. 
Tabela 2. Componentes do balanço de energia pelos métodos das correlações turbulentas $(E C)$ e da razão de Bowen (RB), em cana-de-açúcar variedade RB 92579, no Submédio São Francisco.

\begin{tabular}{lccccccc}
\hline Fases & (Rn-G) & $\boldsymbol{H}_{\boldsymbol{E C}}$ & $\boldsymbol{\lambda} \boldsymbol{E}_{\boldsymbol{E C}}$ & $\frac{\boldsymbol{\lambda} \boldsymbol{E}_{\boldsymbol{E C}}}{\boldsymbol{R \boldsymbol { n } - \boldsymbol { G }}}$ & $\boldsymbol{H}_{\boldsymbol{R} \boldsymbol{B}}$ & $\boldsymbol{\lambda} \boldsymbol{E}_{\boldsymbol{R} \boldsymbol{B}}$ & $\frac{\boldsymbol{\lambda} \boldsymbol{E}_{\boldsymbol{R} \boldsymbol{B}}}{\boldsymbol{R \boldsymbol { n } - \boldsymbol { G }}}$ \\
\hline F1 & $14,5 \pm 0,9$ & $5,4 \pm 1,6$ & $4,3 \pm 0,7$ & 0,29 & $3,5 \pm 0,4$ & $11,4 \pm 0,9$ & 0,79 \\
F2 & $15,9 \pm 0,3$ & $3,2 \pm 0,1$ & $9,9 \pm 0,4$ & 0,62 & $6,7 \pm 0,3$ & $10,2 \pm 0,3$ & 0,64 \\
F3 & $13,0 \pm 0,2$ & $1,8 \pm 0,1$ & $9,4 \pm 0,3$ & 0,72 & $3,4 \pm 0,2$ & $10,0 \pm 0,2$ & 0,77 \\
F4 & $14,5 \pm 0,5$ & $5,4 \pm 0,3$ & $1,1 \pm 0,1$ & 0,08 & $7,8 \pm 0,4$ & $7,5 \pm 0,5$ & 0,52 \\
\hline Ciclo & $14,5 \pm 0,5$ & $3,95 \pm 0,5$ & $9,6 \pm 0,4^{*}$ & $0,66^{*}$ & $5,3 \pm 0,4$ & $9,8 \pm 0,5$ & 0,68 \\
\hline
\end{tabular}

*Valores compreendendo o período das fases F2 e F3, considerado como mais representativo para o método EC por apresentar maior quantidade de dias com medidas obtidas.

comumente encontrado na literatura. Wilson et al. (2002) avaliaram o fechamento do balanço de energia em 50 períodos de um ano em 22 localidades, com períodos de observações entre 1 e 4 anos em cada localidade, e encontraram valores variando entre 15 e $30 \%$ de desigualdade entre $(H+\lambda E)_{E C}$ e $(R n-G)$, em todas localidades.

Os métodos EC e BERB apresentaram as partições da energia disponível $(R n-G)$ de maneira diferente. A Tabela 2 sumariza os valores dos fluxos médios diários por fase de desenvolvimento, bem como a fração evaporativa média diária para cada fase e para o ciclo.

Quando se analisa a fração evaporativa, verificou-se que as fases e destacaram-se como mais representativas do ciclo produtivo da cultura por condicionarem maior número de dias com medidas válidas. Ambos os métodos apresentaram valores semelhantes, embora o método tenha produzido valores ligeiramente inferiores, indicando menores valores de $\lambda E_{E C}$ com relação ao método BERB. Contudo, ambos os métodos confirmaram que a maior parte da energia disponível ao sistema foi convertida em evapotranspiração atual (ETa) (Tabela 2). Verificou-se na fase $\mathrm{F} 1$ que os valores de $\lambda E_{E C}$ foram muito pequenos, enquanto os de $\lambda E_{R B}$ apresentaram-se mais elevados do que os obtidos nas outras fases. Em F4, a suspensão da irrigação condicionou impacto sobre a fração evaporativa, uma vez que a energia disponível foi particionada quase que por igual entre $H_{R B}$ e $\lambda E_{R B}$ (Tabela 2).

Na Figura 2 estão apresentadas as densidades de fluxos obtidas pelos métodos BERB (a) e de EC (b) determinados ao longo do período de estudo. Verificou-se que os valores de $\lambda E_{R B}$ mostraram-se superiores aos valores de $H_{R B}$, com exceção para períodos compreendidos entre 23/12/2010 e 07/01/2011 em que ocorreram reduções bruscas nos valores de $\lambda E_{R B}$ e elevação dos valores de $H_{R B}$.

$O$ método EC também apresentou valores de $\lambda E$ superiores aos de $H$ na maior parte do ciclo produtivo da cultura, com exceção para o início e final do ciclo, em que os valores de $H_{E C}$ mostraram-se superiores aos de $\lambda E_{E C}$, em que, na fase $\mathrm{F} 1 \mathrm{o}$ valor médio do fluxo de $H$ foi ligeiramente superior ao de $\lambda E$ (Tabela 2). Comportamento similar foi constatado para F4, em que a diferença entre estes fluxos apresentou-se mais acentuada. Os maiores valores de erro padrão de estimativa da média obtidos para as fases F1 e F2 quando comparados com os dos outros períodos de desenvolvimento da cultura, indicam que as variações entre os valores dos fluxos de calor sensível e de calor latente obtidos pelos dois métodos na fase inicial e final do ciclo de cultivo.

Comparando-se os dois métodos (Figura 2A, 2B e 2C), observou-se que os valores de $H_{R B}$ foram predominantemente mais elevados do que $H_{\mathrm{EC}}$, até por volta dos $180 \mathrm{DAC}$, quando ocorreu o tombamento característico da cultura, o que proporcionou o aumento da distância entre os psicrômetros do sistema BERB e o dossel da vegetação. Como consequência, ocorreu uma redução nos valores dos fluxos de $H_{R B}$, tornando-os ligeiramente inferiores aos de $H_{E C}$, mas que voltou a se elevar em $\mathrm{F} 4$, quando ocorreu a suspensão da irrigação para maturação fisiológica da cultura. Esta redução pode ser decorrente da diminuição do IAF decorrente do tombamento que, por sua vez, promove redução na quantidade de água evapotranspirada.

Considerando-se os valores médios diários obtidos ao longo do ciclo de cultivo da cultura, verificou-se que a soma $\mathrm{H}+\lambda E_{E C}$ mostrou-se cerca de $25 \%$ inferior à $\mathrm{H}+\lambda E_{R B}$, uma vez que o método força o fechamento do balanço entre os fluxos de energia e a energia disponível (Figura 1B). Por outro lado, comparando-se os fluxos de calor sensível, observou-se que o método $E C$ desfavorece o cálculo dos valores de $H$, uma vez que $H_{\mathrm{FC}}$ foi cerca de $58 \%$ inferior ao de $H_{R B}$ (Figura 3). Essa discrepância, certamente, pode ser atribuída a distorções no fluxo do vento, que são causadas pelo corpo dos sensores e pelas estruturas de montagem do sistema quando o vento sopra em direções diferentes daquela da direção predominante.

Quando se compara os valores de $\lambda E$ determinados por ambos os métodos, verificou-se que o valor de $\lambda E_{E C}$ foi inferior ao de $\lambda E_{R B}$ em apenas $19 \%$, implicando numa diferença de $6 \%$ para menos na comparação entre $H^{+} \lambda E_{E C}$ e $H+\lambda E_{E C}$ ), indicando que os valores obtidos apresentaram boa representatividade da energia disponível e que, portanto, são viáveis para obtenção da evapotranspiração $(E T C)$ e, consequentemente, do coeficiente de cultivo $(K c)$ 


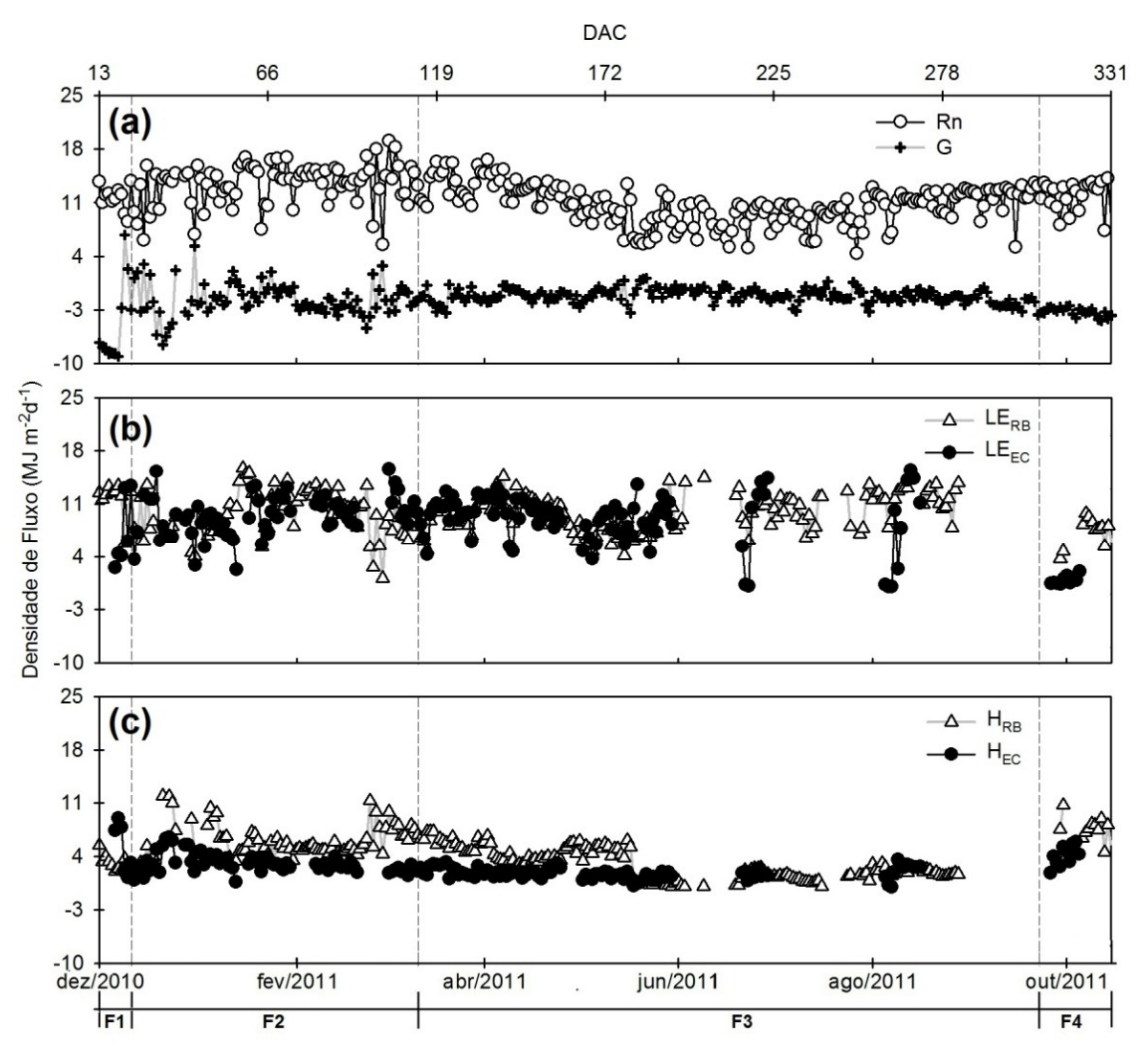

Figura 2. Totais diários dos fluxos de energia determinados na cana-de-açúcar irrigada por gotejamento subsuperficial no Submédio do Vale São Francisco. (a) Saldo de radiação $(R n)$ e fluxo de calor no solo $(G)$; (b) Fluxo de calor latente () e (c) Fluxo de calor sensível (H) determinados pelos métodos das correlações turbulentas (EC) e razão de Bowen (BERB), Juazeiro-BA.

da cultura. Teixeira e Bastiaanssen (2012) verificaram subestimativa de $18 \%$ dos valores de $\lambda E_{E C}$ com relação aos de $\lambda E_{R B^{*}}$

A Figura 4 mostra a variação da evapotranspiração diária para a cana-de-açúcar (a), ao longo do seu ciclo de cultivo, obtida pelos dois métodos, bem como a evapotranspiração de referência (b). Constatou-se que os valores de ETcRB variaram entre 1,5 e 7,4 mm dia ${ }^{-1}$ ao longo do ciclo, enquanto os valores de ETcEC variaram entre 1,2 e 7,1 mm $\operatorname{dia}^{-1}$.

Para a primeira fase de desenvolvimento da cultura (F1), os valores de ETCRB foram superiores aos de ETcEC, variando entre 4,8 e 5,5 mm dia-1, e 1,3 a 4,0 $\mathrm{mm} \mathrm{dia}^{-1}$, com valor médio de $5,1 \pm 0,23$ e $2,2 \pm 1,56 \mathrm{~mm} \mathrm{dia}^{-1}$, respectivamente. Essa diferença acentuada pode ser decorrente da grande diferença entre os níveis de medida dos distintos métodos. Constatou-se também que o valor de ETcRB mostrou-se bastante superior ao obtido por Silva et al. (2012) que utilizando sistema BERB, constatou para essa fase, valor médio de 3,0 $\mathrm{mm}_{\text {dia-1 }}$ para a mesma variedade, em cultivo de cana-de-açúcar irrigada por sulco. Essa fase é caracterizada pela brotação das gemas e pelo início de desenvolvimento da planta, de modo que, a quantidade de vapor d'água transferido para a atmosfera é oriundo, majoritariamente, da evaporação a partir da superfície do solo por se encontrar praticamente desnudo.

$O$ período entre o final de F1 e o início de F2 foi marcado por eventos de precipitação de volume muito pequeno. No período inicial de F2, ETcRB e ETcEC e mostraram tendência semelhante, tendo os valores menores sido obtidos no início, mas que tenderam a se elevar até os 80 DAC, quando então as condições de nebulosidade influenciaram de modo marcante a energia disponível e, consequentemente, os valores de ETc. Os valores médios constatados para esta fase oscilaram entre 2,1 a 7,4 $\mathrm{mm} \mathrm{dia}^{-1}$ e 1,3 a 6,8 mm dia $^{-1}$ para ETcRB e ETcEC respectivamente, com médias de $4,1 \pm 1,22$ e $3,7 \pm 1,06 \mathrm{~mm} \mathrm{dia}^{-1}$, respectivamente (Figura 4).

Valores semelhantes foram constatados na fase seguinte (F3), com ETcRB variando de 1,7 a 7,1 $\mathrm{mm} \mathrm{dia}^{-1}$ (média de 4,1 $\left.\pm 0,99 \mathrm{~mm} \mathrm{dia}^{-1}\right)$ e ETcEC variando entre 1,2 e 7,1 mm $\mathrm{dia}^{-1}$ (média de 3,8 $\pm 1,12 \mathrm{~mm} \mathrm{dia}^{-1}$ ). Essa fase corresponde ao período de máximo crescimento vegetativo e que pode apresentar maiores valores de ETc quando comparados com os das demais fases, condição esta não constatada no presente estudo. Os baixos valores de ETc mostraram-se relativamente reduzidos nessa fase em decorrência da coincidência com o período de inverno, em que a disponibilidade de energia é reduzida, as temperaturas são mais amenas, o que resulta numa menor demanda evapotranspirométrica da atmosfera. 

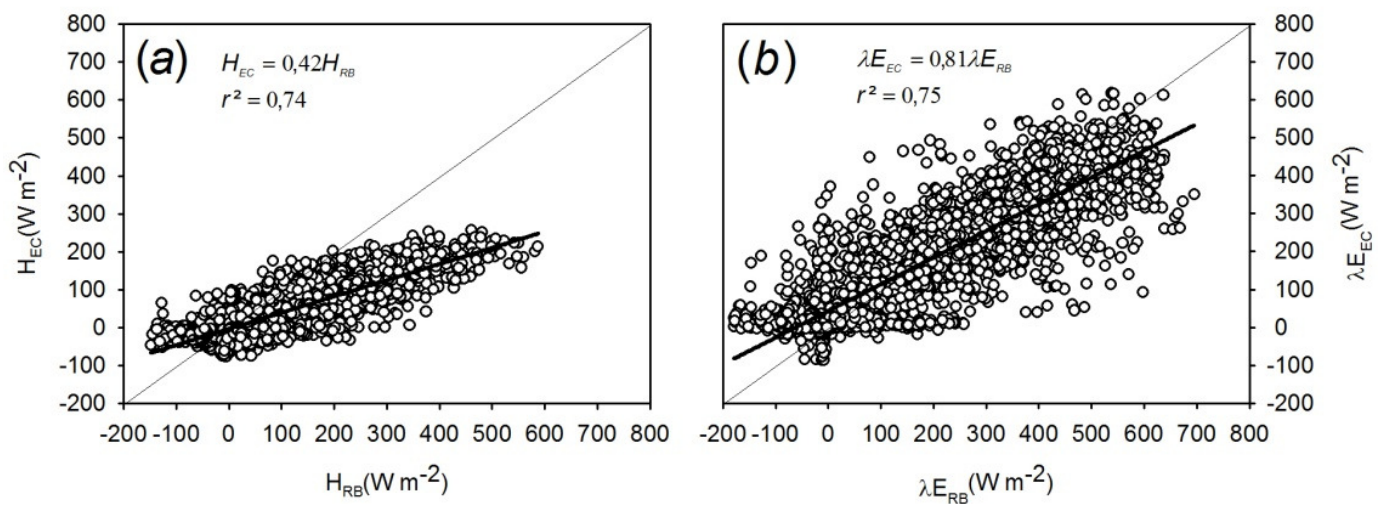

Figura 3. Relação entre os fluxos de calor sensível (a) e calor latente (b) determinados pelos métodos das correlações turbulentas $(E C)$ e da razão de Bowen (BERB) para a cana-de-açúcar irrigada por gotejamento subsuperficial no Submédio do Vale São Francisco

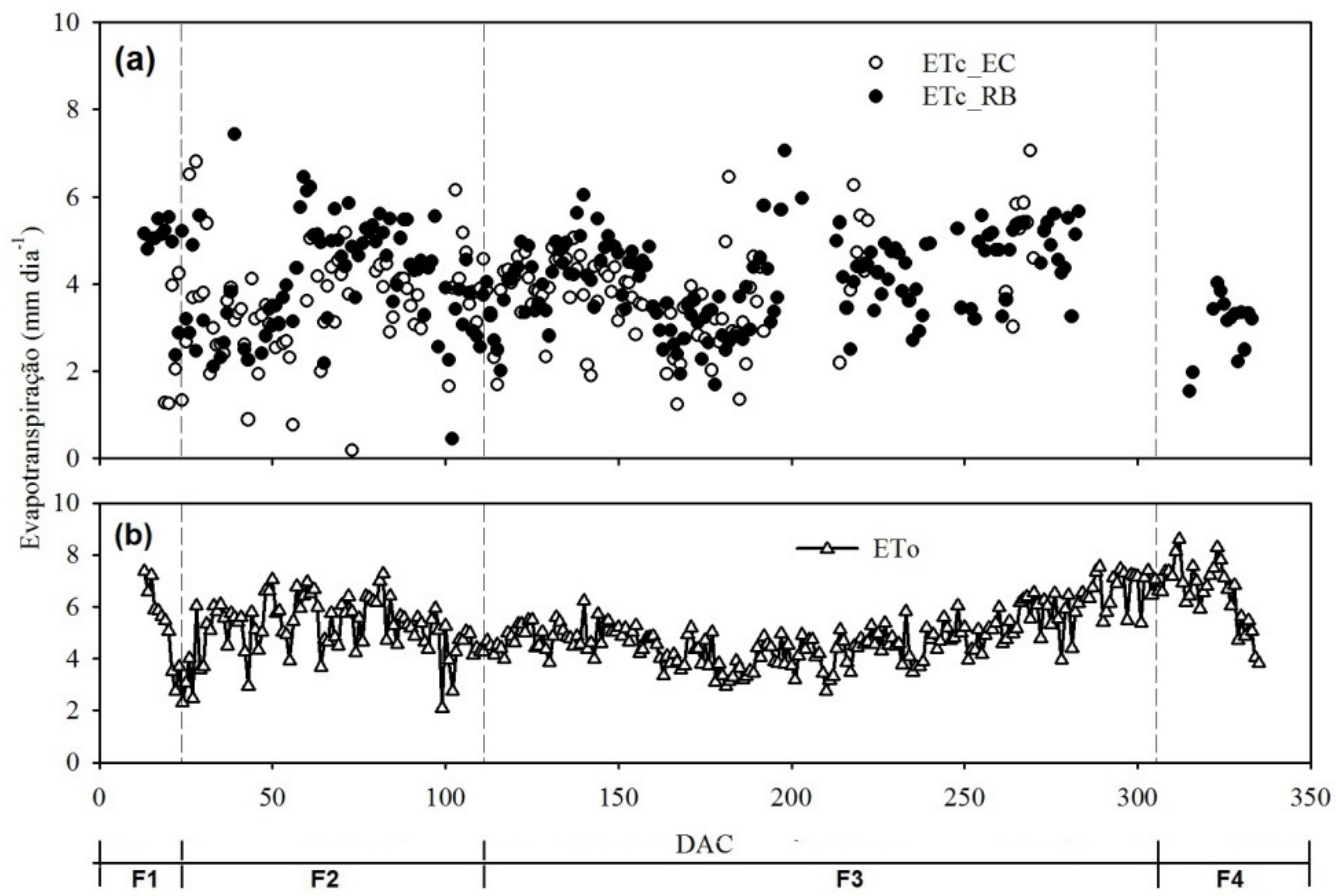

Figura 4. Evapotranspiração de cultivo ao longo do ciclo da cana-de-açúcar, obtida pelos métodos BERB (ETCRB) e EC (ETcEC), Juazeiro-BA.

A fase F4 é caracterizada pela suspensão da irrigação para condicionar a maturação fisiológica da cultura, o que implica numa redução da ETc, como se pode observar com os valores de ETcRB, que mostraram um declínio acentuado (Figura 4), apresentando valores que oscilaram entre $1,4 \mathrm{~mm}$ e 4,0 mm e média de 3,0 $\pm 0,7 \mathrm{~mm}$. Em termos gerais, a evapotranspiração média diária obtida pelo método eddy covariance apresentou-se inferior à obtida pelo método do balanço de energia com base na razão de Bowen, cujas mé-

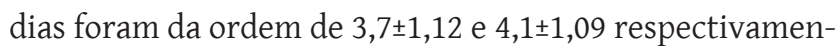
te.

Analisando-se o comportamento do coeficiente de cultivo obtido para a cultura da cana-de-açúcar ao longo do seu ciclo produtivo, observou-se para a fase 1 que os va- lores variaram entre 0,23 e 1,13 para o método EC e entre 0,70 e 0,86 para o método $B E R B$, cujas médias foram de $0,54 \pm 0,51$ e de $0,75 \pm 0,07$ respectivamente (Figura 5). Vale salientar que as medições e armazenamento dos dados se iniciaram aos 13 DAC, quando foi finalizada a instalação completa dos equipamentos e que eventos de precipitação ocorreram ao final da fase 1 . Verificou-se que os valores de $K c_{E C}$ médio obtido foi próximo aos valores determinados por Inman Bamber \& McGlynchey $(2003)(0,4)$ e pela FAO (Allen et al, 1998) $(0,40)$, enquanto o valor médio de $K C_{R B}$ foi bastante superior,devido à grande diferença entre os valores de ETCEC e ETCRB nessa fase de desenvolvimento.

No entanto, os valores de $K C_{R B}$ foram mais próximo do valor determinados por Silva et al. (2012), que foi da or- 


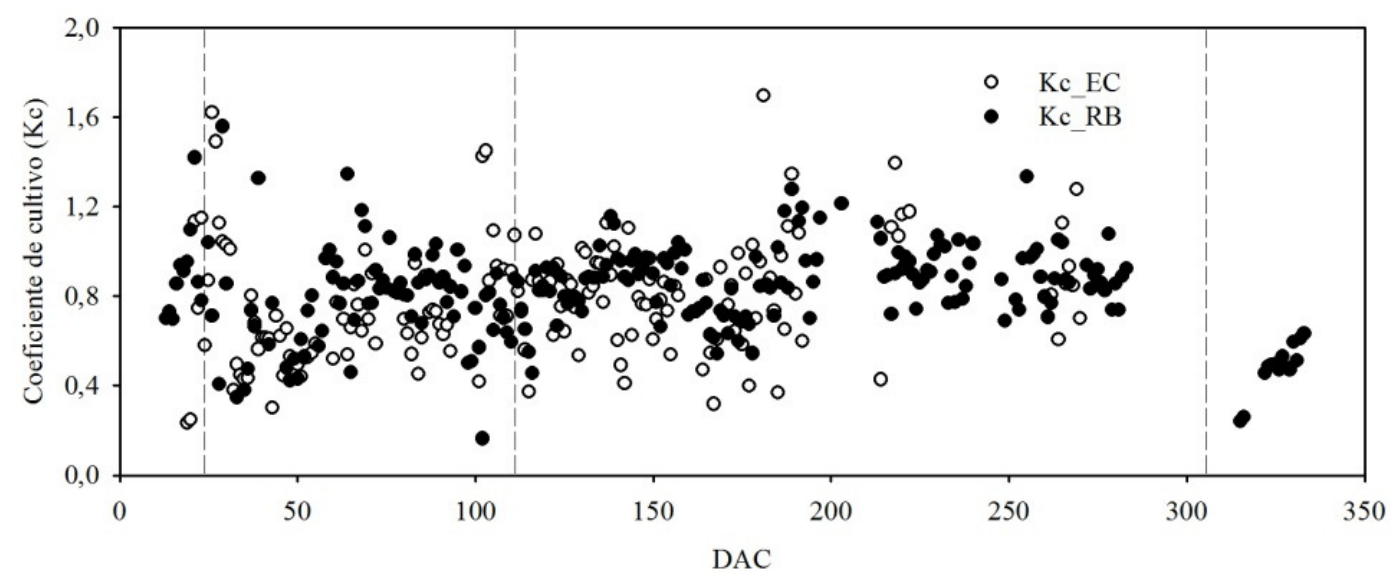

Figura 5. Valores médios diários do coeficiente de cultivo $(K c)$ da cana-de-açúcar obtido pelos métodos Eddy Covariance e Balanço de Energia - Razão de Bowen, Juazeiro-BA.

dem de 0,65 para a mesma variedade, sob condições climáticas semelhantes e utilizando o método BERB.Vale salientar que o Kc médio para a fase inicial da cana-de-açúcar sob condições de alta frequência de irrigação ou períodos chuvosos pode aumentar substancialmente podendo alcançar valores de 1,2 .

Constatou-se na fase F2, que é caracterizada pelo estabelecimento e perfilhamento da cultura, que os valores de $K c_{E C}$ foram mais elevados que os da fase F1, variando entre 0,38 e 1,62, com média de $0,74 \pm 0,26$. Os valores de $K c_{R B}$ apresentaram elevação menos acentuada com relação aos valores da fase 1 variando entre 0,35 e 1,56, com média de $0,79 \pm 0,22$ de modo que as médias dos dois métodos apresentaram valores muito próximos (Figura 5).

Na fase F3, que é caracterizada pelo máximo crescimento da cultura, de março a setembro, foi constatada maior equidade na determinação dos valores de $K C$ determinados pelos dois métodos com relação à fase anterior, com KcEC variando entre 0,31 e 1,70, apresentando média de $0,83 \pm 0,24$, que apresentou-se ligeiramente inferior à média do $\operatorname{KcRB}(0,87 \pm 0,14)$. Nessa fase, com a cultura plenamente estabelecida e o solo totalmente encoberto, a $E T$ depende, predominantemente da transpiração das plantas, que foi influenciada pelas características climáticas predominantes. Pôde-se constatar que os valores apresentados apresentaram-se bastantes inferiores aos valores informados no boletim 56 da FAO $(1,25)$. Em região semiárida da Tailândia, Watanabe et al. (2004) encontraram um valor médio de 1,10 para a fase de máximo crescimento da cana-de-açúcar, o mesmo constatado por Silva et al. (2012) sob condições climáticas do semiárido brasileiro, porém o período de máximo crescimento da cultura coincidiu com a estação mais quente e seca nos dois estudos. Do mesmo modo, Gonçalves (2010) obteve Kc médio de 1,03 para a fase de máximo crescimento da cana-de-açúcar irrigada por gotejo subsuperficial, no estado do Ceará, plantada no mês de julho.
Na última fase de desenvolvimento (F4), que corresponde à fase de maturação da cana-de-açúcar, observou-se que o valor médio de KcRB apresentado pela cultura foi de $0,52 \pm 0,1$ (variação entre 0,24 e 0,78), enfatizando o estresse hídrico ao qual a cultura foi submetida. A FAO recomenda, para esse estágio, $K c$ de 0,75 para cultivos sem estresse hídrico, bem manejados, em climas sub-úmidos (minima $\approx 45 \% ; \approx 2 \mathrm{~m} \mathrm{~s}^{-1}$ ). Não foi possível obter o $\mathrm{KcEC}$ para essa fase devido à ocorrência de problemas com a aquisição de dados.

Com os valores de KC definidos para cada fase de desenvolvimento da cana-de-açúcar, foram feitas extrapolações dos valores de ETc para os dias excluídos (considerados inválidos), bem como para os dias no início e final do ciclo, nos quais não foram realizadas medições, com exceção para a fase $\mathrm{F} 4$, pelo método $E C$, devido à falha no armazenamento de dados.

Logo, obtiveram-se a ETC acumulada para o ciclo de cultivo, bem como para cada uma das fases de desenvolvimento da cana (Figura 6). A ETcRB acumulada para todo o ciclo de cultivo foi da ordem de $1478 \mathrm{~mm}$, inferior à demanda atmosférica (ETo=1805 mm), bem como aos 1687 $\mathrm{mm}$ que entraram no sistema por precipitação e irrigação $(P+I)$. Da lâmina de água total que entrou no sistema, 1497 $\mathrm{mm}$ foram aplicados por irrigação e apenas $190 \mathrm{~mm}$ foram devidos à precipitação pluviométrica.

Analisando-se a demanda de água da cana em cada uma das fases de desenvolvimento (Figura 6), constatou-se que na fase F1 os valores acumulados de ETCRB e de ETCEC foram da ordem de $103 \mathrm{~mm}$ e $69 \mathrm{~mm}$, enquanto a lâmina de água que entrou no sistema por P+I foi da ordem de 127 $\mathrm{mm}$, dos quais $106 \mathrm{~mm}$ foram devidos à irrigação e apenas $21 \mathrm{~mm}$ foram oriundos de precipitação pluviométrica. Esses valores indicam que a demanda de água pela cultura foi atendida nessa fase, havendo, inclusive, um pequeno excesso na lâmina aplicada.

A fase F2 de desenvolvimento da cana apresentou va- 


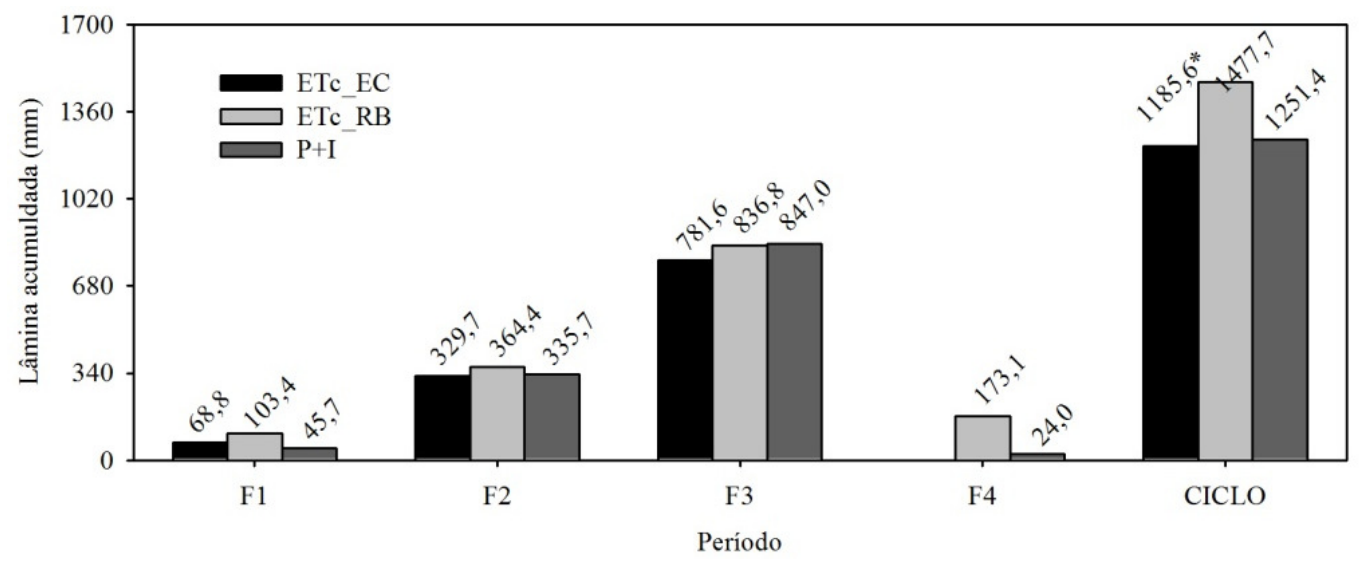

Figura 6. Lâminas de entrada $(P+I)$ e saída ( $E T c E C$ e ETcRB) de água em cada fase de desenvolvimento da cultura e total acumulado ao final do ciclo, Juazeiro-BA.

lores de consumo de água semelhantes entre os métodos $B E R B$ e $E C$ tendo acumulado $364 \mathrm{~mm}$ e $330 \mathrm{~mm}$ nessa ordem. No entanto, a lâmina de água que entrou no sistema por irrigação foi de $508 \mathrm{~mm}$ que mais $113 \mathrm{~mm}$ de precipitação totalizaram uma lâmina de água de $621 \mathrm{~mm}$ nesta fase, excesso que promoveu a saturação do solo que, combinada à elevada umidade relativa do ar constatada nessa fase (média de 76,4\%), possivelmente contribuiu para os baixos valores de ETC e Kc obtidos. Vale ressaltar que a umidade relativa do ar média para cada fase de desenvolvimento foi inferior a $70 \%$ apenas na fase $\mathrm{F} 4$.

$\mathrm{Na}$ fase de máximo crescimento vegetativo (F3), constatou-se que $P+I$ foi na ordem de $894 \mathrm{~mm}$ ( $P=43 \mathrm{~mm}$ e $I=$ $851 \mathrm{~mm}$ ) enquanto a lâmina de água requerida determinada pelo método $B E R B$ foi $837 \mathrm{~mm}$, e pelo método $E C, 787$ $\mathrm{mm}$.

A fase final do ciclo de cultivo, caracterizada pela suspensão da irrigação, apresentou demanda de água de 173 $\mathrm{mm}$, determinada pelo método $B E R B$, uma vez que, nesse período, o método $E C$ apresentou problemas no armazenamento de dados.

No entanto, o processo de maturação fisiológica, que visa o aumento no acúmulo de sacarose nas plantas, induzido pelo estresse pode ter sido afetado devido à presença de água disponível no solo, acumulada ao longo das fases F1, F2 e F3 nas quais a lâmina de água aplicada excedeu a lâmina demandada pela cultura (Figura 6).

Vale salientar que o Kc utlizado na irrigação da cana-de-açúcar ao longo do ciclo do presente estudo foi determinado por Silva et al. (2012), os quais, com exceção da fase F1, são superiores aos constatados no presente estudo, justificando, portanto a lâmina de água de irrigação superior à lâmina de água demandada pela cultura.

\section{Conclusões}

Constatou-se que o método das correlações turbulentas subestimou os valores dos fluxos de energia e, por consequência, a evapotranspiração da cultura, quando comparados com os fluxos obtidos pelo método da razão de Bowen;

Ambos os métodos revelaram que a maior parte da energia disponível ( $\sim 67 \%)$ foi convertida em evapotranspiração real;

A evapotranspiração média diária da cultura diferiu entre os dois métodos, apresentando valores de 3,6 e 4,1 $\mathrm{mm} \mathrm{d}^{-1}$ para os métodos EC e BERB, resultando em um requerimento hídrico total de $1478 \mathrm{~mm}$.

Ao longo do ciclo de cultivo da cana-de-açúcar, os valores do coeficiente de cultura foram da ordem de 0,54 e de 075 na fase F1, 0,74 e 0,79 para a fase F2, 0,83 e 0,87 na fase F3 para os métodos EC e BERB, respectivamente, e 0,52 para a fase $\mathrm{F} 4$ pelo método $\mathrm{BERB}$.

A lâmina de água aplicada ao longo do ciclo produtivo foi superior à evapotranspiração da cultura devido ao Kc utilizado para realizar as irrigações em cada fase de desenvolvimento ter sido maior do que o obtido no presente estudo.

\section{Referências}

ALLEN, R. G. et al. Evapotranspiration information reporting: I. Factors governing measurement Accuracy. Agricultural Water Management, Amsterdam, v. 98, p. 899-920, 2011.

ALLEN, R. G. et al. Crop evapotranspiration: guidelines for computing crop water requirements. Rome, Italy: FAO, 1998. Irrigation and Drainage Paper 56.

GONÇALVES, F. M. Evapotranspiração e coeficientes de cultivo da cana-de-açúcar irrigada por gotejamento subsuperficial. 2010. 65 f. Dissertação (Mestrado), Universidade Federal do Ceará, Fortaleza. 
INMAN-BAMBER, N. G.; MCGLINCHEY, M. G. Crop coefficients and wateruse estimates for sugarcane based on long-term Bowen ratio energy balance measurements. Field Crops Research, Amsterdam, v. 83, p. 125-138, 2003.

INMAN-BAMBER, N. G.; SMITH, D. M. Water relations in sugarcane and response to water deficits. Field Crops Research, Amsterdam, v. 92, p. 185-202, 2005.

MARIN, F. Evapotranspiração e transpiração máxima em cafezal adensado. 2003. Tese (Doutorado) 134 f. Escola Superior de Agricultura “Luiz de Queiroz" Universidade de São Paulo, Piracicaba.

PEREZ, P. J.; CASTELLVI, F.; IBAÑEZ, M.; ROSELL, J. I. Assessment of reliability of Bowen ratio method for partitioning fluxes. Agricultural and Forest Meteorology, Amsterdam, v. 97, p. 141-150, 1999.

SILVA, L. D. B.; FOLEGATTI, M. V.; VILLA NOVA, N. A. Evapotranspiração do capim Tanzânia obtida pelo método de razão de Bowen e lisímetro de pesagem. Engenharia Agrícola, Botucatu, v. 25, n. 3, p. 705-712, 2005.

SILVA, T. G. F. et al. Variação do balanço de radiação e de energia da cana-de-açúcar irrigada no semiárido brasileiro. Revista Brasileira de Engenharia Agrícola e Ambiental, Campina Grande, v. 15, n. 2, p. 139-147, 2011.

SILVA, T. G. F. et al. Requerimento hídrico e coeficiente de cultura da cana-de-açúcar irrigada no semiárido brasileiro. Revista Brasileira de Engenharia Agrícola e Ambiental, Campina Grande, v. 16, n. 1, p. 64-71, 2012.
STEDUTO, P.; HSIAO, T. C. Maize canopies under two soil water regimes. I. Diurnal patterns of energy balance, carbon dioxide flux, and canopy conductance. Agricultural and Forest Meteorology, Amsterdam, v. 89, p. 169-184, 1998.

TEIXEIRA, A. H. de C.; BASTIAANSSEN, W. G. M.; Five methods for interpret Field measurements of energy fluxes over a micro-sprinklerirrigated mango orchard. Irrigation Science, Amsterdam, v. 30, p. 13-28, 2012.

TEIXEIRA, A. H. C.; BASTIAANSSEN, W. G. M.; BASSOI, L. H. Crop water parameters of irrigated wine and table grapes to support water productivity analysis in the Sao Francisco river basin, Brazil. Agricultural Water Management, Amsterdam, v. 94, p. 31-42, 2007.

WATANABE, K. et al. Changes in seasonal evapotranspiration, soil water content, and crop coefficients in sugarcane, cassava, and maize fields in Northeast Thailand. Agricultural Water Management, Amsterdam, v. 67, p. 133-143, 2004.

WILSON, K. B. et al. Energy balance closure at FLUXNET sites.

Agricultural and Forest Meteorology, Amsterdam, v. 113, p. 223-243, 2002.

REFERENCIAÇÃO CARMO, J. F. A. do; MOURA, M. S. B. de; SILVA, T. G. F. da; SOUZA, L. S. B. de; LEITÃo, M. de M. V. B. R. Balanço de energia e coeficiente de cultura da cana-de-açúcar por dois métodos micrometeorológicos. Agrometeoros, Passo Fundo, v.25, n.1, p.101-111, 2017.

Declaração: os trabalhos estão sendo publicados nesse número de AGROMETEOROS (v.25, n.1, ago 2017) conforme foram aceitos pelo XX Congresso Brasileiro de Agrometeorologia, realizado de 14 a 18 de agosto de 2017, em Juazeiro, BA e Petrolina, PE, sem revisão editorial adicional da revista. 


\title{
Energy balance and crop coefficient of the sugarcane by two micrometeorological methods
}

\author{
José Francisco Alves do Carmo ${ }^{1}$, Magna Soelma Beserra de Moura ${ }^{2(*)}$, Thieres George Freire da Silva ${ }^{3}$, \\ Luciana Sandra Bastos de Souza ${ }^{3}$, Mario de Miranda Vilas Boas Ramos Leitao ${ }^{4}$ \\ ${ }^{1}$ Mestre, CPGEA, UNIVASF, Juazeiro-BA, j.francarmo@gmail.com \\ ${ }^{2}$ Pesquisadora, Embrapa Semiárido, Petrolina-PE, magnamoura@embrapa.br \\ ${ }^{3}$ Professor, UFRPE/UAST, Serra Talhada-PE, thieres_freire@yahoo.com.br, sanddrabastos@yahoo.com.br; 4Professor, PPGEA, UNIVASF, Juazeiro-BA, \\ mario.miranda@univasf.edu.br \\ ${ }^{(*)}$ Corresponding author
}

\section{ARTICLE INFO}

Article history:

Received 16 June 2017

Accepted 10 August 2017

\section{Index terms:}

eddy covariance

evapotranspiration

Submedio of the São Francisco Valley

Bowen ratio

Saccharum spp

\section{ABSTRACT}

The aim of this study was to determine the evapotranspiration of sugarcane under subsurface drip irrigation in semi-arid conditions of the lower basin of the Valley of the São Francisco using the methods of turbulent correlations and Bowen ratio. Therefore, the densities of latent and sensible heat fluxes were measured in culture throughout the production cycle during the period November 2010 to October 2011. These results showed that the method presented EC underestimation of the energy available by $26 \%$, and the latent heat flux by $19 \%$ compared to the method of Bowen. Evaporation fraction of the daily values ranged from 0.66 (turbulent correlation method) and 0.68 (Bowen method) reveals that most of the available energy is converted to ETA. Average daily evapotranspiration cultivation differ between the two methods, with values of 3.6 and $4.1 \mathrm{~mm} \mathrm{~d}^{-1}$ for EC and RB methods, resulting in a total water requirement of $1478 \mathrm{~mm}$. The sugarcane crop coefficient values obtained were 0.54 and 075 in the initial phase, 0.83 and 0.87 in the phase of maximum growth of the EC and BERB methods, respectively, and 0.52 for phase end, determined by the BERB method.

Disclaimer: papers are published in this issue of AGROMETEOROS (v. 25, n.1, aug 2017) as accepted by the XX Congresso Brasileiro de Agrometeorologia, held August 14-18, 2017 in Juazeiro, Bahia and Petrolina, Pernambuco, Brazil, without further revision by editorial board. 